\title{
Anesthetic Management of a Pregnant Patient with CSVT for Emergency LSCS and Craniotomy
}

\author{
Authors \\ Dr Naresh.V, Dr Sudhakar. L, Dr Chandra Sekhar C.N. \\ Department of Anaesthesiology and Pain Medicine \\ Yashoda Hospital, Somajiguda, Hyderabad
}

\begin{abstract}
Cerebral sinus venous thrombosis is a rare phenomenon seen in pregnant woman and one of the major causes of stroke with high mortality. Cerebral venous thrombosis with intracranial hemorrhage has a poor outcome than with CSVT alone. Here, we report a case of a 27 year old female primi-gravida with 32 weeks of gestational age diagnosed as CSVT with hemorrhage, underwent LSCS followed by craniotomy in the same setting, managed successfully.
\end{abstract}

Keywords: CSVT, CVT, Pregnancy, LSCS, Craniectomy.

\section{Introduction}

Cerebral Sinus Venous thrombosis is one of the causes of hemorrhagic stroke in pregnancy, which needs to be diagnosed and treated early to prevent adverse outcomes for mother and fetus. Management of CSVT in pregnancy requires multidisciplinary approach involving neurosurgeon, obstetrician, anesthesiologist and intensivist for the best possible outcome. Here, we report anesthetic and perioperative management of a 27-year-old female patient who developed CSVT complicated by Intracranial hemorrhage with midline shift taken up for emergency LSCS followed by Craniotomy, managed successfully with a positive outcome.

\section{Case Report}

A 27-year-old pregnant woman came to Emergency Department with complaints of headache, weakness in Right upper and lower limbs, followed by slurred speech. Patient is a primi gravida with 32 weeks of gestational age. She is a known hypothyroid on thyronorm 75 micrograms daily. She had a history of PCOD, treated with ovulation induction drugs 2 years ago. She was also on ecosprin $75 \mathrm{mg}$ since one year.

On initial assessment, her GCS was $\mathrm{E}_{4} \mathrm{~V}_{5} \mathrm{M}_{6}$, but weakness in right upper and lower limbs was present with a power of $2 / 5$. Her pupils were $2 \mathrm{~mm}$ bilateral, reacting to light. She was hemodynamically stable at the time of presentation.

After initial treatment with antiepileptics, mannitol and steroids, MRI + MRV was done which showed a venous infarct with hemorrhage in Left Frontal lobe and suspicious thrombus in anterior part of superior sagittal sinus. (Fig 1 and Fig. 2) Patient is diagnosed as Cerebral venous sinus thrombosis. 


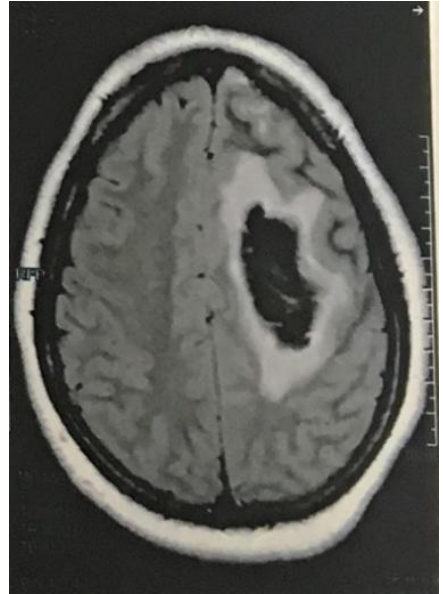

Fig.1. MRI showing Left Superior Frontal lobe hematoma

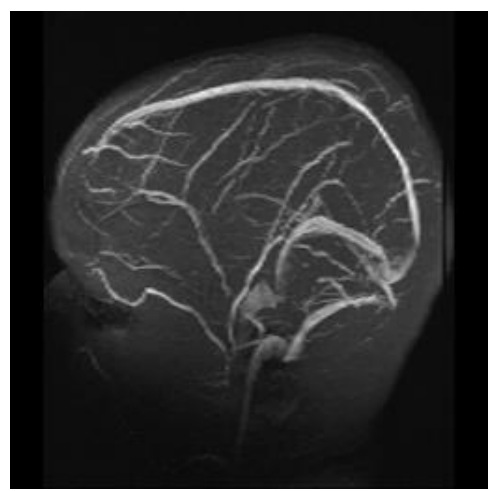

Fig.2. MRV showing loss of signal in anterior $3^{\text {rd }}$ of superior sagittal sinus and adjacent left frontal cortical veins - likely thrombosis

Patient was initially treated in ICU for CSVT. Multidisciplinary approach involving obstetrician, neurosurgeon, intensivist and anesthesiologist consultations were taken. Anticipating emergent cesarean section, betamethasone was given for lung maturity of fetus.

On $3^{\text {rd }}$ day of admission, patient became irritable and deviation of mouth to right side was noted. Subsequently she became drowsy. EEG was done, which showed intermittent slowing. She further became drowsy with deteriorating GCS E2V2M5.

MRI was done which showed LEFT frontal bleed of $6 \times 4 \mathrm{~cm}$ with midline shift of $8 \mathrm{~mm}$ towards right was noted, which has increased compared to previous MRI.(Fig.3)

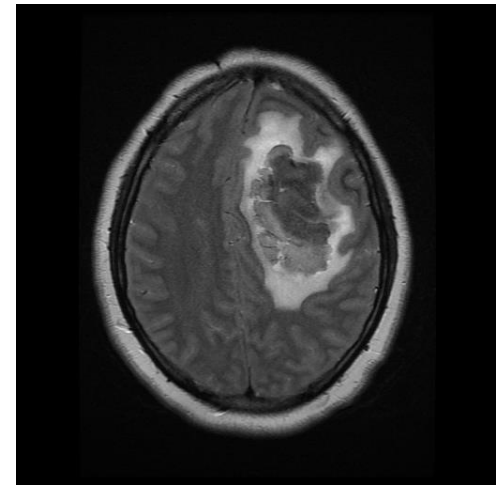

Fig.3. MRI showing Left Frontal hematoma with midline shift on to right side.

In view of impending herniation, patient was posted for emergency LSCS followed by decompressive craniotomy.

Preoperative anesthesiologist evaluation was done. Patient was drowsy, incoherent. On examination, patient had Right hemiplegia. Pupils were unequal with Rt. pupil measuring $5 \mathrm{~mm}$ and Left pupil measuring $3 \mathrm{~mm}$, but both were reacting to light. Examination of other systems was normal. Patient was otherwise hemodynamically stable.

Patient attendants were counselled regarding plan of surgery and anesthesia and associated risks and benefits and the need for post operative mechanical ventilation.

Patient attendants after discussions and deliberations for about $4 \mathrm{hrs}$ gave a consent for the emergency LSCS and craniotomy. Since there was time delay between the MRI and the consent from the attendants, neurosurgeon wanted a CT scan to be done which is better diagnostic tool than MRI to delineate the extent of bleed and also to rule out PCA infarct seen in conditions of raised ICP and midline shift and which if present is a poor prognostic feature.

So, it was planned to do a CT scan soon after the LSCS.

After confirming the NBM status, high risk consent was taken. Inj ranitidine $50 \mathrm{mg}$ i.v was given in ICU.

General anesthesia was given. Patient was induced with $100 \mathrm{mg}$ propofol IV. Rapid sequence intubation done using rocuronium $50 \mathrm{mg}$ with Sellicks maneuver. Patient was intubated with 7.0 cuffed OETT. General anesthesia was maintained 
with oxygen $50 \%$ with air and Sevoflurane as inhalational agent to maintain MAC just above 0.7. Muscle relaxation was maintained by subsequent bolus doses of atracurium. Patient delivered a live male baby, weighing $2 \mathrm{~kg}$ within 4 minutes of induction. Baby had mild respiratory distress and was shifted to NICU for observation. Fentanyl 100 micrograms was given just after the delivery of baby. 15 units of oxytocin was added to iv fluid after delivery. Oxytocin infusion was continued throughout the surgery and post operatively. Blood loss was estimated to be around $500 \mathrm{ml}$.

After the surgery patient was shifted for CT scan on OETT and bains cirucit ventilation. Standard monitoring was continued throughout the transport and during CT scan. CT brain plain showed large left fronto-parietal bleed of size $67 \mathrm{x}$ $43 \times 52$ with edema and midline shift of $9 \mathrm{~mm}$ towards right side. There is no intraventricular extension and PCA infarct is ruled out.( Fig.4)

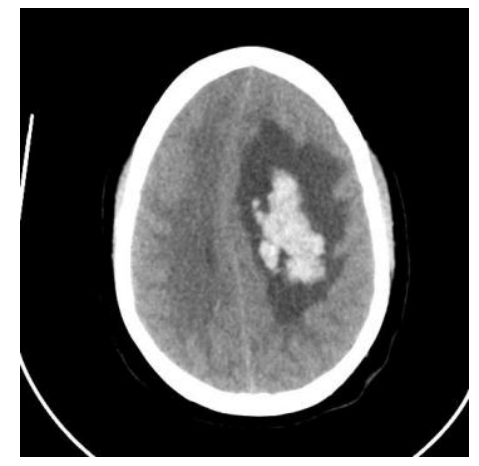

Fig 4. CT scan showing left frontal hematoma and midline shift

Patient was transferred to operating room immediately for emergency decompressive craniectomy. Ventilation was continued and maintained with sevoflurane and oxygen $40 \%$ with air. Fentanyl 100 micrograms was given as intraop analgesia. Muscle relaxation was maintained using atracurium $50 \mathrm{mg}$ bolus and intermittent bolus doses. Inj mannitol 40 grams and frusemide $20 \mathrm{mg}$ was given intraoperatively to reduce intracranial pressure. Blood loss was estimated to be $700 \mathrm{ml} \& 1$ unit of FFP and 1 unit of Packed RBC was given intraoperatively to compensate for ongoing blood losses.

$1500 \mathrm{ml}$ of crystalloids were given during craniectomy. Urine output was monitored hourly and was adequate. Surgery went on for $90 \mathrm{~min}$ and was unventful. Patient was hemodynamically stable.

In view of low preoperative GCS, patient was planned to be ventilated electively and was shifted to ICU for further management. Patient was sedated and paralysed to prevent ventilator dyssynchrony and raise in intracranial pressure. Measures to prevent DVT, bed sores, gastric ulcers were taken care of.

CT scan on post operative day 1 showed reduction and reversal of midline shift and ischemic changes in internal capsule. But there was slight increase in edema.

Patient was extubated on POD 3 after patient regained consciousness and was obeying commands. Power in the right upper and lower limbs has not improved still. After observation for one more day, patient was shifted to ward on POD 4 and was discharged on post operative day 6.

\section{Discussion}

Pregnancy is a hypercoagulable state and has a high risk of developing thrombotic complications. Incidence of stroke in pregnancy is estimated to be around 25- 34 per 100,000 deliveries of which hemorrhage accounts for 13 of 34 strokes identified $^{1,2}$. Common causes of hemorrhage in pregnancy include rupture of A Malformation, hypertension and Cerebral Venous Thrombosis. CVT undiagnosed or untreated, leads to increased venous pressure in brain, cerebral edema and intracranial hemorrhage.

Risk factors for CVT include drugs like OCPs, Steroids, HRT and treatment for cancer.

Patients typically present with headache as commonest symptom. Some may present with cranial nerve palsies. All the cranial nerves may be involved except for the olfactory nerve. Patients may also present with seizures which favours the diagnosis of venous thrombosis rather than arterial thrombus.

Our patient presented with headache and weakness in the right upper and lower limbs, aphasia and later on also developed seizures. 
Neurological deficit in this patient may be related to hemorrhagic infarct.

Early diagnosis and prompt decision for choice of treatment are important prognostic factors for the patient.MRI in combination with MRV is the investigation of choice for diagnosis of CVT.

Anesthetic technique and management plays a significant role for a better outcome of mother and fetus. Anesthesiologist requires thorough knowledge of physiological changes of pregnancy, its implications on intracranial pressure, effects of drugs and ventilation on fetus.

It is important to decide when to deliver the fetus, as the patient may any time land up for an emergency craniotomy. Sudden neurological deterioration requires securing of airway in emergency. It is to be kept in mind that every pregnant woman in third trimester may have a difficult airway due to edema and structural changes in pregnancy. There is also a high risk of aspiration due to delayed gastric emptying.

Mechanical ventilation and maneuvers to reduce ICP may compromise the fetal oxygenation. Osmotic diuresis with mannitol has been shown to cause fetal hypovolemia. Hyperventilation to wash out carbon dioxide and reduce ICP causes respiratory alkalosis, shifting the ODC curve to left and decreases oxygen delivery to fetus.

Decision to deliver the fetus depends on gestational age and the fetal maturity. If the Gestational age is advanced, it is recommended to deliver the fetus to prevent adverse pregnancy outcomes $^{3}$.

In our case it was initially planned to continue pregnancy and manage CVT conservatively. But due to sudden neurological deterioration, it was decided to do an emergency craniotomy, which prompted the delivery of fetus also in the same setting.

Craniotomy in a third trimester pregnant woman may lead to adverse outcomes like fetal hypoxia and premature delivery ${ }^{4,5}$. It is a difficult task to manage the patient, if she required a prolonged post operative ventilation, as there is a need for continuous and intense fetal monitoring, in addition to neurological and ICP monitoring.
There is always a risk of pulmonary infections, hypoxia, acidosis, hypovolemia leading to fetal hypoxia requiring emergent $\mathrm{LSCS}^{6}$.

So, in our patient it was decided to do a LSCS first, immediately followed by decompressive craniotomy.

Regional anesthesia was avoided in this patient as there was a risk of herniation. General anesthesia is preferred choice of anesthesia in this patient, in view of patient being drowsy and raised ICP being contraindication for regional anesthesia.

Goals of anesthetic management is to avoid hypoxia, hypercarbia and hypotension. Measures to reduce ICP, like hyperventilation and use of osmotic diuretics should be used in caution. Hemodynamic stability is very important to maintain cerebral and uteroplacental perfusion. Measures to prevent hypotension include appropriate fluid administration, avoiding aortocaval compression and use of vasopressors if required $^{7}$.

Intubation stress response should be avoided, as it increases ICP.

All inhalational anesthetics cause uterine muscle relaxation. Isoflurane appears to be least potent uterine relaxant of the volatile anesthetics ${ }^{8}$. Minimum alveolar concentration of 0.5 for sevoflurane and desflurane is considered safe without any fear of uterine atony ${ }^{9}$. Nitrous oxide is avoided, as it raises ICP.

There were few case reports where combined caesarean section and craniotomy were done previously. Bisri et al, reported a case of a pregnant woman with 32 weeks of gestational age in fetal distress and low GCS ${ }^{10}$. CT scan showed left temporoparietal intracranial mass with midline shift. She underwent LSCS followed by craniotomy for tumor evacuation.

Another case report by khurana et al, presented a 25 year old female with 33 weeks gestational age underwent craniotomy for glioma excision and cesarean section in the same sitting ${ }^{11}$.

In summary, it is a challenging task to manage a pregnant woman in third trimester presenting for emergency craniotomy, where in the decision, when to operate and deliver the fetus is important 
and needs proper coordination between the obstetrician, neurosurgeon, anesthesiologist and intensivist for a safe maternal and fetal outcome.

\section{References}

1. Grear K.E, Bushnell, C.D . Stroke and pregnancy: Clinical presentation, evaluation, treatment and epidemiology. Clin Obstet Gynaecol. 2013; 56(2): 350 -359 .

2. Jaigobin C, Silver FL. Stroke and pregnancy. Stroke. 2000; 31:2948-51.

3. Le LT, Wendling A. Anesthetic management for cesarean section in a patient with rupture of a cerebellar arteriovenous malformation. J Clin Anesth. 2009 Mar ;21(2):143-8.

4. Lapinsky SE, Rojas-Suarez JA, Crozier TM, Vasquez DN, Barrett N, Austin K, et al. Mechanical ventilation in critically-ill pregnant women: a case series. Int J Obstet Anesth. 2015 Nov; 24(4):323-8.

5. Subramanian R, Sardar A, Mohanaselvi S, Khanna P, Baidya DK. Neurosurgery and pregnancy. J Neuroanaesthesiol Crit Care 2014;1:166-72.

6. Kazuyoshi Aoyama, P Gareth Seaward and Stephen E Lapinsky. Fetal outcome in the critically ill pregnant woman. Crit Care. 2014; 18(3): 307.

7. Wang LP, Paech MJ. Neuroanesthesia for the pregnant woman. Anesth Analg. 2008 Jul;107(1):193-200.

8. Yoo KY, Lee JC, Yoon MH, Shin $\mathrm{MH}$, Kim SJ, Kim YH, et al. The effects of volatile anesthetics on spontaneous contractility of isolated human pregnant uterine muscle: a comparison among sevoflurane, desflurane, isoflurane, and halothane. Anesth Analg. 2006 Aug;103(2):443-7.

9. Yildiz K, Dogru K, Dalgic H, Serin IS, Sezer Z, Madenoglu H, et al. Inhibitory effects of desflurane and sevoflurane on oxytocin-induced contractions of isolated pregnant human myometrium. Acta Anaesthesiol Scand. 2005 Oct; 49(9):1355-9.

10. Bisri DY, Wullur C, Bisri T. Anaesthetic management for combined emergency caesarean section and craniotomy tumour removal. J Neuroanaesthesiol Crit Care. 2017; 4:53-6.

11. Khurana, T, Taneja, B, \& Saxena K. N. Anesthetic management of a parturient with glioma brain for caesarean section immediately followed by craniotomy. J Anaesthesiol Clin Pharmacol. 2014. jul Sep. 30(3), 397-399. 